\title{
NOTA
}

\section{PONTO DE EFEITO SALINO NULO DE LATOSSOLOS DA MICROBACIA CHICO NUNES, MATO GROSSO ${ }^{(1)}$}

\author{
Elaine Arruda Oliveira Coringa ${ }^{(2)}$ \& Oscarlina Lúcia dos Santos Weber ${ }^{(3)}$
}

\begin{abstract}
RESUMO
A utilização de atributos eletroquímicos tem sido sugerida por muitos autores como forma de caracterizar o complexo coloidal do solo e servir como índices de avaliação de certas propriedades relacionadas à fertilidade e à nutrição das plantas e à pedogênese, possibilitando diferenciar os solos de região para região. No presente trabalho foram avaliadas as relações entre o ponto de efeito salino nulo (PESN) e as propriedades químicas de três Latossolos de uma toposseqüência pertencente à Microbacia Chico Nunes, Mato Grosso. Amostras superficiais e subsuperficiais dos solos foram submetidas às caracterizações química, mineralógica e eletroquímica. $O$ teor de $\mathrm{C}$ orgânico, de maneira inversa, e o teor de Al vinculado a formas mal cristalizadas, de forma direta, explicaram a quase totalidade da variação do PESN nos solos estudados. Os valores do PESN determinados por meio de titulação potenciométrica mostraram-se bem correlacionados com os valores de ponto de carga zero (PCZ) estimados a partir de valores de pH medidos em solução de $\mathrm{KCl} 1 \mathrm{~mol} \mathrm{~L}^{-1}(\mathrm{pH} \mathrm{KCl})$ e em água (pH $\mathrm{H}_{2} \mathrm{O}$ ) por meio da equação $\mathrm{PCZ}=2 \mathrm{pH} \mathrm{KCl}-\mathrm{pH} \mathrm{H}_{2} \mathrm{O}$, reiterando sua utilidade na estimativa de valores de PESN de solos tropicais com carga variável. Do mesmo modo, o índice $\Delta \mathrm{pH}\left(\Delta \mathrm{pH}=\mathrm{pH} \mathrm{KCl}-\mathrm{pH} \mathrm{H}_{2} \mathrm{O}\right)$ mostrou-se altamente correlacionado com a diferença PESN - pH $\mathrm{H}_{2} \mathrm{O}$, comprovando a aplicabilidade desse índice na estimativa do sinal e da magnitude da carga líquida superficial de solos altamente intemperizados.
\end{abstract}

Termos de indexação: cargas elétricas superficiais, dupla camada difusa, solos de Cerrado.

\footnotetext{
(1) Parte da Tese de Mestrado do primeiro autor apresentada ao Programa de Pós-graduação em Agricultura Tropical da Universidade Federal de Mato Grosso - UFMT. Trabalho apresentado no XXX Congresso Brasileiro de Ciência do Solo, Recife (PE). Recebido para publicação em setembro de 2005 e aprovado em agosto de 2007.

${ }^{(2)}$ Doutoranda em Agricultura Tropical - Recursos Naturais, Faculdade de Agronomia e Medicina Veterinária, Universidade Federal de Mato Grosso - FAMEV/UFMT. CEP 78060-900 Cuiabá (MT). E-mail: elainecoringa@terra.com.br

(3) Pesquisadora em Solos e Nutrição de Plantas, Departamento de Solos e Engenharia Rural, FAMEV/UFMT. E-mail: oscsanwb@ufmt.br
} 


\title{
SUMMARY: POINT OF NULL SALINE EFFECT OF OXISOLS IN THE MICROBASIN CHICO NUNES - MATO GROSSO, BRAZIL
}

\begin{abstract}
The use of electrochemical attributes has been suggested by a number of authors to characterize the colloidal soil compound and to serve as evaluation indices of certain properties related to plant fertility and nutrition and to pedogenesis, underlying regional soil differentiation. In this study, the relations between the point of null saline effect (PESN) and chemical properties of three Oxisols of a toposequence in the microbasin Chico Nunes, Mato Grosso were evaluated. Surface and subsurface samples were submitted to chemical, mineralogical and electrochemical characterization. The organic carbon, inversely, and the oxalate-Al oxides, directly, explained almost the entire variation of the PESN in the studied soils. The PESN values determined by means of potentiometric titration were well correlated with the values of point of zero charge (PCZ), calculated from $\mathrm{pH}$ values measured in $\mathrm{KCl} 1 \mathrm{~mol} \mathrm{~L} \mathrm{~L}^{-1}$ solution $\left(\mathrm{pH}_{\mathrm{KCl}}\right)$ and in water $\left(\mathrm{pH}_{\mathrm{H} 2 \mathrm{O}}\right.$ ) by means of the equation $P C Z=p H_{K C l}-p H_{H 2 O}$, reinforcing its usefulness in estimating PESN values of tropical soils with variable charge. Likewise, the index $\mathrm{DpH}=p \mathrm{H}_{\mathrm{KCl}}-\mathrm{pH} \mathrm{H}_{\mathrm{H} O \mathrm{O}}$ ) was highly correlated with difference PESN - $p H_{H 2 O}$, proving the applicability of this index in the estimate of the signal and the magnitude of the surface liquid charge of highly weathered soils.
\end{abstract}

Index terms: Cerrado soils, , double diffuse layer, surface electric charge.

\section{INTRODUÇÃO}

Os solos bem drenados das regiões tropicais úmidas são intensamente intemperizados e dominados por minerais com carga variável, o que lhes confere baixa capacidade de troca catiônica (CTC), a qual varia com o pH (Agbenin \& Raij, 1999). Muitos estudos sobre as características dos solos tropicais úmidos intemperizados têm relatado o estabelecimento das relações entre os seus atributos eletroquímicos, químicos e mineralógicos, o que facilita a compreensão dos fenômenos de adsorção/dessorção de íons no solo.

Dentre os atributos eletroquímicos, o de maior destaque para solos intemperizados das regiões tropicais é o ponto de efeito salino nulo (PESN), utilizado na descrição de fenômenos decorrentes da dupla camada elétrica de interfaces reversíveis (Sakurai et al., 1989; Alleoni \& Camargo, 1994a,b; Zhang \& Zhao, 1997) e na determinação de vários atributos pedológicos (Fontes et al., 2001).

O PESN tem importante impacto nas propriedades de carga do solo, pois a diferença entre o PESN e o pH do solo determina o sinal e a magnitude da carga variável presente. Quando o $\mathrm{pH}$ do solo for menor que o PESN, os sítios de carga variável tornam-se predominantemente carregados positivamente, facilitando a retenção aniônica. Por outro lado, se o pH do solo for maior que o PESN, eles desenvolvem, na sua maioria, carga negativa, favorecendo a troca catiônica (Uehara \& Gillman, 1981)

Assim, pode-se afirmar que a densidade de carga na superfície pode ser alterada em solos com carga variável pela aplicação orientada de fertilizantes e corretivos, aumentando significativamente a CTC e, portanto, a capacidade produtiva desses solos. Uma outra característica importante acerca do PESN do solo é que ele corresponde ao ponto químico de máxima estabilidade, onde o potencial de superfície é nulo (Sposito, 1989). Assim, solos ricos em sílica ou orgânicos, com PESN baixo, tendem a ser mais ácidos que os solos oxídicos altamente intemperizados, com alto PESN (Nascimento et al., 1988).

O valor do PESN do solo depende da composição mineralógica deste e da presença de matéria orgânica humificada. Os óxidos de Fe apresentam ponto de carga zero entre 7 e 9 (Schwertmann \& Taylor, 1989), e os óxidos de $\mathrm{Al}$, entre 8 e 9,2 (Hsu, 1989). Muitos autores têm observado relação direta entre os valores de PESN e a composição mineralógica da fração argila de horizontes subsuperficiais, e em solos oxídicos o valor do PESN é próximo ao valor do PCZ de óxidos de Fe e Al puros (Raij \& Peech, 1972; Gallez et al., 1977), denotando semelhança entre o PCZ do mineral predominante da fração argila e o PESN do solo. Por isso, esta medida eletroquímica é utilizada muitas vezes como forma de avaliar o grau de intemperismo do solo e, conseqüentemente, do desenvolvimento pedológico, visando a sua aplicação à taxonomia dos solos.

A importância da determinação desse atributo eletroquímico em solos vai desde a previsão do desenvolvimento pedogenético (Hendershot \& Lavkulich, 1978) à definição de toposseqüência e cronosseqüência (Hendershot et al., 1979; Parker et al., 1979), bem como do comportamento do solo quanto à dissolução de minerais (Chorover \& Sposito, 1995). Portanto, estudos realizados acerca do PESN em solos 
envolvem desde métodos de determinação até práticas de manejo adequadas, com a finalidade de alterar o seu valor para aumentar a capacidade produtiva dos solos tropicais.

Nesse contexto, o estudo de seqüências de solos tem-se mostrado eficiente na avaliação da influência dos fatores de formação dos solos, podendo-se observar contrastes entre as características condicionadas pela mineralogia, topografia e hidrologia de cada superfície. Coscione et al. (2005) sugeriram o uso de alguns atributos químicos e eletroquímicos do solo como essenciais para descrever os processos físico-químicos que ocorrem simultaneamente com as transformações morfológicas em toposseqüências de solos intemperizados.

O objetivo deste trabalho foi avaliar as relações do PESN com atributos químicos de Latossolos de uma toposseqüência localizada na Microbacia Chico Nunes, Estado de Mato Grosso.

\section{MATERIAL E MÉTODOS}

Amostras foram coletadas nos horizontes A e B de três Latossolos de uma toposseqüência da Microbacia Hidrográfica Chico Nunes, situada a $48 \mathrm{~km}$ do município de Primavera do Leste, Mato Grosso. Os solos foram classificados do topo para a base da vertente, correspondendo a uma diferença de nível de $33 \mathrm{~m}$, em Latossolo Vermelho-Amarelo distrófico típico (solos 1 e 2) e em Latossolo Amarelo distrófico típico (solo 3) (Embrapa, 1999).

Após secas ao ar, destorroadas e passadas em peneira de $2 \mathrm{~mm}$ de abertura de malha, as amostras dos horizontes $\mathrm{A} \mathrm{e}_{\mathrm{w}}$ diagnóstico foram caracterizadas quanto à granulometria, aos valores de $\mathrm{pH}$ medidos em solução de $\mathrm{KCl} 1 \mathrm{~mol} \mathrm{~L}^{-1}(\mathrm{pH} \mathrm{KCl})$ e em água ( $\mathrm{pH} \mathrm{H} \mathrm{H}_{2} \mathrm{O}$ ), ao teor de $\mathrm{C}$ orgânico e de óxidos dos ataques sulfúrico $\left(\mathrm{Fe}_{2} \mathrm{O}_{3}\right.$ e $\left.\mathrm{Al}_{2} \mathrm{O}_{3}\right)$ e alcalino $\left(\mathrm{SiO}_{2}\right)$, com os quais se calcularam os índices $\mathrm{Ki}$ e $\mathrm{Kr}$, e aos teores de Fe e Al extraíveis em ditionito-citrato-bicarbonato de sódio (DCB) a $75^{\circ} \mathrm{C}$ (Mehra \& Jackson, 1960) $\left(\mathrm{Fe}_{\mathrm{d}} \mathrm{e}\right.$ $\mathrm{Al}_{\mathrm{d}}$ ) e em solução ácida de oxalato de amônio (OXA) (Schwertmann, 1964) $\left(\mathrm{Fe}_{0}\right.$ e $\left.\mathrm{Al}_{o}\right)$. Todas as determinações supramencionadas foram realizadas adotando-se os métodos compilados e adaptados por Camargo et al. (1986), e os elementos foram dosadas por espectrofotometria de absorção atômica.

O PESN foi determinado a partir de dados de titulação potenciométrica (Costa et al., 1984). Foram adicionados aos solos $20 \mathrm{~mL}$ de solução de $\mathrm{KCl}$ em três concentrações $\left(0,001 ; 0,01 ;\right.$ e $\left.0,1 \mathrm{~mol} \mathrm{~L}^{-1}\right)$ e volumes de $\mathrm{HCl} 0,1 \mathrm{~mol} \mathrm{~L}^{-1}$ e de $\mathrm{NaOH} 0,1 \mathrm{~mol} \mathrm{~L}^{-1}$, correspondentes às concentrações de 0,$5 ; 1,0 ; 1,5 ; 2,0$; 3,$0 ; 4,0$; e $6,0 \mathrm{cmol}_{\mathrm{c}} \mathrm{dm}^{-3} \mathrm{de} \mathrm{H}^{+}$e 1,0 e $2,0 \mathrm{cmol}_{\mathrm{c}} \mathrm{dm}^{-3}$ de $\mathrm{OH}^{-}$. As suspensões de solo foram agitadas e colocadas em repouso por $24 \mathrm{~h}$, e posteriormente foi determinado o $\mathrm{pH}$ com um $\mathrm{pHmetro}$ digital. As curvas da titulação potenciométrica para cada concentração de $\mathrm{KCl}$ foram construídas plotando-se as quantidades de $\mathrm{H}^{+}$ou $\mathrm{OH}^{-}$adsorvidos pela amostra contra os valores de $\mathrm{pH}$ atingidos pelas suspensões em equilíbrio. $\mathrm{O}$ valor do $\mathrm{PESN}$ foi considerado o valor de $\mathrm{pH}$ do ponto de interseção das curvas de titulação obtidas para cada concentração do eletrólito. Para construção das curvas de titulação potenciométrica e tratamento dos dados obtidos, foi utilizado o programa computacional PESN 1.0, desenvolvido por Alves et al. (2002). Adicionalmente, o ponto de carga zero (PCZ) das amostras foi estimado por meio da equação $\mathrm{PCZ}=$ $2 \mathrm{pH} \mathrm{KCl}-\mathrm{pH} \mathrm{H} \mathrm{H}_{2} \mathrm{O}$ (Keng \& Uehara, 1974).

A composição mineralógica da fração argila dos horizontes $\mathrm{B}_{\mathrm{w}}$ dos solos foi determinada por difratometria de raios $\mathrm{X}$ após remoção da matéria orgânica com peróxido de hidrogênio a $30 \%$ a $50{ }^{\circ} \mathrm{C}$ e desferrificação. A desferrificação da fração argila consistiu de quatro extrações sucessivas com solução de ditionito -citrato-bicarbonato de sódio (DCB) a $75^{\circ} \mathrm{C}$, segundo Mehra \& Jackson (1960). Uma parte da fração argila desferrificada foi submetida à saturação com Mg e a outra à saturação com K. Quanto à concentração dos óxidos de Fe na fração argila, utilizou-se solução concentrada de $\mathrm{NaOH}$ à temperatura de $100{ }^{\circ} \mathrm{C}$, com posterior centrifugação e secagem do resíduo em estufa a $105^{\circ} \mathrm{C}$, por $12 \mathrm{~h}-$ Norrish \& Taylor (1961), modificado por Kampf \& Schwertmann (1982). Foi estimado o valor da substituição isomórfica por Al na estrutura da hematita (Schwertmann, 1988; Muggler et al., 2001) e da goethita (Schwertmann \& Carlson, 1994) nos horizontes $\mathrm{B}_{\mathrm{w}}$. A relação $\mathrm{Hm} / \mathrm{Hm}+\mathrm{Gt}$ foi calculada com base na intensidade dos picos da hematita no plano 104 e da goethita no plano 110 dos difratogramas de raios X, segundo Jones (1981).

Os resultados experimentais e calculados foram submetidos a análises de correlação linear, visando avaliar suas associações. O efeito dos atributos dos solos sobre o PESN foi avaliado por meio da regressão linear múltipla, adotando-se o procedimento stepwise de seleção automática de variáveis independentes. Para compensar o pequeno número de pontos experimentais, fixou-se o nível de significância para entrada e permanência de cada variável independente na equação de regressão em valores menores que $2 \%$ de probabilidade. A existência de multicolinearidade na equação de regressão foi avaliada por meio do cálculo do fator de inflação da variância (SAS, 1994).

\section{RESULTADOS E DISCUSSÃO}

A classe textural dos solos estudados enquadrouse nas classes franco-argilo-arenosa a franco-arenosa, com aumento de argila em profundidade. Os solos apresentaram diminuição do $\mathrm{pH}_{\mathrm{H} 2 \mathrm{O}}$ em profundidade e seus valores foram maiores que o $\mathrm{pH} \mathrm{KCl} \mathrm{nos}$ 
horizontes $\mathrm{A}$; o inverso foi observado em todos os horizontes $\mathrm{B}_{\mathrm{w}}$ (Quadro 1), indicando presença de carga líquida superficial positiva no horizonte subsuperficial.

O teor de C orgânico total (COT) apresentou-se maior nos horizontes superficiais dos solos, decrescendo em profundidade, independentemente da posição do perfil no relevo.

O teor de Fe no extrato sulfúrico aumentou em subsuperfície (Quadro 1), e o maior valor foi obtido no horizonte $\mathrm{B}_{\mathrm{w}}$ do perfil 1 , possivelmente devido à maior quantidade de Fe cristalino nas feições mais profundas, como demonstrado pela razão $\mathrm{Fe}_{\mathrm{d}} / \mathrm{Fe}_{\mathrm{t}}$, que variou de 0,60 a 0,70, condizente com a natureza latossólica dos solos (Lacerda et al., 2000; Barreal et al., 2003; Inda Júnior \& Kämpf, 2003; Chuan-Wen et al., 2004; Pai et al., 2007). O decréscimo do $\mathrm{Fe}_{\mathrm{T}}$ ao longo da vertente pode indicar maior mobilidade do $\mathrm{Fe}$ ao longo da toposseqüência, como resultado da drenagem dos solos (Ghidin et al., 2006). Os teores de $\mathrm{Al}$ foram proporcionalmente dominantes no extrato sulfúrico de todos os solos.

Os valores de $\mathrm{Ki}$ e $\mathrm{Kr}$, em geral, foram baixos e semelhantes para todos os solos da toposseqüência (0,88 e 0,77 em média, respectivamente) (Quadro 1), indicando uniformidade da mineralogia da fração argila com predomínio de caulinita e óxidos de Fe e Al. Essa afirmação se baseia na classificação de Latossolos muito intemperizados proposta por Resende \& Santana (1988), em que os solos são classificados como caulinítico sesquioxídico (perfil 1) e caulinítico não-sesquioxídico (perfis 2 e 3). Esses índices

\section{Quadro 1. Atributos químicos e eletroquímicos dos solos da toposseqüência}

\begin{tabular}{|c|c|c|c|c|c|c|}
\hline \multirow{2}{*}{ Atributo } & \multicolumn{2}{|c|}{ Perfil 1 - LVAd } & \multicolumn{2}{|c|}{ Perfil 2 LVAd } & \multicolumn{2}{|c|}{ Perfil 3 LAd } \\
\hline & A & $\mathbf{B}_{\mathbf{w}}$ & A & $\mathbf{B}_{\mathbf{w}}$ & A & $\mathbf{B}_{\mathbf{w}}$ \\
\hline Profundidade (m) & $0-0,2$ & $1,02-1,70$ & $0-0,2$ & $0,92-1,78^{+}$ & $0-0,19$ & $1,43-1,72^{+}$ \\
\hline Teor de argila $\left(\mathrm{g} \mathrm{kg}^{-1}\right)$ & 302 & 391 & 331 & 353 & 294 & 382 \\
\hline $\mathrm{pH} \mathrm{H}_{2} \mathrm{O}$ & 6,5 & 5,3 & 6,1 & 5,1 & 6,0 & 5,5 \\
\hline $\mathrm{pH} \mathrm{KCl}$ & 5,7 & 5,9 & 5,2 & 5,6 & 5,1 & 6,0 \\
\hline $\mathrm{COT}^{(1)}\left(\mathrm{g} \mathrm{kg}^{-1}\right)$ & 20,9 & 5,9 & 18,2 & 3,2 & 17,0 & 4,1 \\
\hline $\mathrm{Fe}_{2} \mathrm{O}_{3}{ }^{(2)}\left(\mathrm{g} \mathrm{kg}^{-1}\right)$ & 45,4 & 57,2 & 40,2 & 51,8 & 37,3 & 47,7 \\
\hline $\mathrm{Al}_{2} \mathrm{O}_{3}{ }^{(2)}\left(\mathrm{g} \mathrm{kg}^{-1}\right)$ & 226,8 & 273,6 & 165,8 & 280,1 & 158,8 & 241,0 \\
\hline $\mathrm{SiO}_{2}{ }^{(2)}\left(\mathrm{g} \mathrm{kg}^{-1}\right)$ & 109,0 & 125,4 & 76,9 & 150,7 & 112,6 & 129,7 \\
\hline $\mathrm{Ki}$ & 0,82 & 0,78 & 0,79 & 0,91 & 1,21 & 0,92 \\
\hline $\mathrm{Kr}$ & 0,72 & 0,69 & 0,68 & 0,82 & 1,05 & 0,81 \\
\hline $\mathrm{Fe}_{\mathrm{d}}^{(3)}\left(\mathrm{g} \mathrm{kg}^{-1}\right)$ & 27,5 & 37,3 & 28,1 & 34,1 & 24,8 & 33,1 \\
\hline $\mathrm{Al}_{\mathrm{d}}^{(3)}\left(\mathrm{g} \mathrm{kg}^{-1}\right)$ & 37,8 & 38,8 & 27,4 & 30,3 & 28,4 & 33,1 \\
\hline $\mathrm{Fe}_{o}^{(4)}\left(\mathrm{g} \mathrm{kg}^{-1}\right)$ & 6,3 & 2,6 & 6,1 & 2,0 & 5,2 & 2,3 \\
\hline $\mathrm{Al}_{0}^{(4)}\left(\mathrm{g} \mathrm{kg}^{-1}\right)$ & 12,6 & 11,8 & 9,7 & 5,1 & 9,3 & 9,2 \\
\hline $\mathrm{Fe}_{o} / \mathrm{Fe}_{\mathrm{d}}$ & 0,23 & 0,07 & 0,22 & 0,06 & 0,21 & 0,07 \\
\hline $\mathrm{Fe}_{\mathrm{d}} / \mathrm{Fe}_{\mathrm{T}}$ & 0,60 & 0,65 & 0,70 & 0,66 & 0,67 & 0,69 \\
\hline $\mathrm{Fe}_{\mathrm{o}} / \mathrm{Fe}_{\mathrm{T}}$ & 0,14 & 0,05 & 0,15 & 0,04 & 0,14 & 0,05 \\
\hline$\Delta \mathrm{pH}$ & $-0,8$ & 0,6 & $-0,9$ & 0,5 & $-0,9$ & 0,5 \\
\hline PESN & 3,9 & 6,2 & 4,0 & 5,8 & 3,9 & 6,0 \\
\hline $\mathrm{PCZ}^{(5)}$ & 4,8 & 6,5 & 4,4 & 6,1 & 4,2 & 6,5 \\
\hline $\mathrm{SI}_{\mathrm{Hm}}{ }^{(6)}\left(\mathrm{mol} \mathrm{mol}^{-1}\right)$ & - & 0,19 & - & 0,19 & - & 0,11 \\
\hline $\mathrm{SI}_{\mathrm{Gt}}{ }^{(7)}\left(\mathrm{mol} \mathrm{mol}{ }^{-1}\right)$ & - & 0,32 & - & 0,37 & - & 0,32 \\
\hline $\mathrm{Hm} / \mathrm{Hm}+\mathrm{Gt}^{(8)}(\%)$ & - & 11 & - & 12 & - & 8 \\
\hline $\mathrm{PESN}-\mathrm{pH} \mathrm{H} \mathrm{H}_{2} \mathrm{O}$ & $-2,6$ & 0,9 & $-2,1$ & 0,7 & $-2,1$ & 0,5 \\
\hline
\end{tabular}

\footnotetext{
(1) Carbono orgânico total. ${ }^{(2)}$ Teores de óxidos totais no extrato sulfúrico $\left(\mathrm{Fe}_{\mathrm{t}}\right){ }^{(3)}$ Teores de óxidos de ferro e alumínio cristalizados. ${ }^{(4)}$ Teores de óxidos de ferro e alumínio mal cristalizados. ${ }^{(5)}$ Ponto de carga zero estimado pela equação PCZ: 2 pH KCl pH $\mathrm{H}_{2} \mathrm{O}$ (Keng \& Uehara, 1974). ${ }^{(6)}$ Substituição isomórfica por Al na estrutura da hematita: $\mathrm{Al}_{\mathrm{Hm}}\left(\mathrm{mol} \mathrm{mol}^{-1}\right)$ : $31,41-62,3 . \mathrm{a}_{0}=$ dimensão a da célula unitária da hematita, em nm, calculada a partir do valor obtido por DRX do espaçamento interplanar referente ao plano 110 da hematita (Schwertmann, 1988; Muggler et al., 2001). (7) Substituição isomórfica por Al na estrutura da goethita: $\mathrm{Al}_{\mathrm{Gt}}\left(\mathrm{mol} \mathrm{mol}^{-1}\right): 14,62-48,3 . \mathrm{c}_{\mathrm{o}}=$ dimensão $\mathrm{c}$ da célula unitária da goetita, em nm, calculada a partir do valor obtido por DRX dos espaçamentos interplanares referentes aos planos 111 e 110 da goetita (Schwertmann \& Carlson, 1994). ${ }^{(8)} \mathrm{Hm} /(\mathrm{Hm}+\mathrm{Gt}): 1 /\left[\mathrm{A}_{\mathrm{Gt} 110} /\left(\mathrm{A}_{\mathrm{Hm} 104} \cdot 0,708\right)\right]+1$ (Jones, 1981).
} 
aumentam à medida que se aproxima da base da toposseqüência, apesar de não ser aumento significativo, o que pode indicar ressilificação dos minerais da fração argila, provocada pelo fluxo de matéria translocada pelo movimento lateral de água para as partes mais baixas da vertente, conforme Canellas et al. (2000). Os maiores valores foram obtidos no horizonte superficial do perfil 3, revelando que este solo é menos intemperizado que os solos suprajacentes. Isso pode ser explicado pela posição do perfil no relevo: partes mais baixas da toposseqüência estão menos sujeitas aos fatores que promovem o intemperismo dos solos, o que contribui para aumento do Ki e Kr. O grau de intemperismo e a ação dos processos específicos de formação determinaram variações significativas nas características dos solos ao longo da toposseqüência, demonstrando que o pedoambiente influencia a estabilidade dos minerais no solo, e as condições de drenagem interna causada pela topografia são um fator crítico na transformação e redistribuição dos minerais de argila (Pai et al., 2007).

Os difratogramas da fração argila desferrificada comprovaram presença de caulinita, gibbsita e caulinita associada à clorita $(0,36$ e $0,35 \mathrm{~nm})$, e com menor intensidade foi detectada a presença de traços de anatásio e minerais $2: 1$, como a mica-ilita, em todas as amostras, tal como observado por Alleoni \& Camargo (1995) em solos ácricos.

Os difratogramas mostraram reflexões de alta intensidade de gibbsita, confirmando o maior grau de intemperização dos solos, demonstrado pelos baixos valores de $\mathrm{Ki}$ e $\mathrm{Kr}, \Delta \mathrm{pH}$ e PESN positivo nos horizontes Bw (Quadro 1). Esses dados sugerem haver maior perda de silica nas partes superiores da toposseqüência e sua concentração nas partes inferiores, com conseqüente influência sobre a mineralogia dos solos. Segundo Ker \& Resende (1990), as condições de percolação lenta de água no transcurso da toposseqüência parecem favorecer o aumento gradual de sílica, a qual pode combinar com o $\mathrm{Al}$, formando a caulinita. Isso está registrado nos difratogramas dos horizontes $\mathrm{B}_{\mathrm{w}}$, onde a intensidade das reflexões de gibbsita $(0,478$ e $0,433 \mathrm{~nm})$ foi maior no perfil 1 , enquanto no perfil 3 houve aumento expressivo da intensidade do pico da caulinita $(0,721$ e $0,358 \mathrm{~nm})$, evidenciando a transformação da caulinita em gibbsita da base para o topo da toposseqüência.

Os difratogramas dos concentrados de óxidos de Fe revelaram a presença de goethita e hematita como óxidos de Fe predominantes na fração argila dos solos da toposseqüência. Verificou-se que o maior grau de substituição do Fe por Al na estrutura cristalina foi atribuído à goethita (Quadro 1), semelhante aos resultados obtidos por Muggler et al. (2001) e Alves (2002) em Latossolos. A $\mathrm{SI}_{\mathrm{Gt}}$ foi 2,13 vezes maior que a $\mathrm{SI}_{\mathrm{Hm}}$ nos horizontes $\mathrm{B}_{\mathrm{w}}$ dos solos, de acordo com Schwertmann \& Kämpf (1985), que mencionam a relação $2: 1$ para as substituições de goethitas e hematitas coexistentes em uma amostra, baseando- se na capacidade teórica da goethita de acomodar duas vezes mais $\mathrm{Al}$ em sua estrutura do que a hematita. Os valores da relação $\mathrm{Hm} /(\mathrm{Hm}+\mathrm{Gt})$ evidenciaram o efeito da drenagem sobre os solos da toposseqüência: valores maiores foram encontrados no topo da vertente, enquanto valores menores foram obtidos no perfil 3. Schwertmann (1988) afirma que a drenagem deficiente provoca efeito anti-hematítico nos solos, por dificultar a desidratação da ferridrita, mineral precursor da hematita.

Mais de $60 \%$ do Fe presente nos solos estava vinculado aos óxidos cristalinos e mal cristalizados $\left(\mathrm{Fe}_{\mathrm{d}}\right)$, enquanto as formas mal cristalizadas de $\mathrm{Fe}\left(\mathrm{Fe}_{\mathrm{o}}\right)$ contiveram, em média, $10 \%$ do Fe total, evidenciando o avançado estádio de intemperismo desses solos. Os teores de $\mathrm{Fe}_{0}$ foram mais elevados no horizonte subsuperficial, possivelmente devido à ocorrência de matéria orgânica em superfície, que possibilita ambiente mais redutor, favorecendo a formação de óxidos de Fe mal cristalizados (Lacerda et al., 2000; Kämpf \& Curi, 2003). Esse fato pode ser visualizado pela relação $\mathrm{Fe}_{\mathrm{o}} / \mathrm{Fe}_{\mathrm{d}}$ muito inferior em subsuperfície, que indica o alto grau de cristalinidade dos óxidos de Fe (Inda Júnior et al., 2005).

Com relação ao $\mathrm{Al}$, verificou-se predomínio das formas cristalinas $\left(15 \%\right.$ em $\mathrm{Al}_{\mathrm{d}}$ ) sobre as mal cristalizadas $\left(5,2 \%\right.$ em $\left.\mathrm{Al}_{0}\right)$, e estas frações de $\mathrm{Al}$ geralmente apresentaram-se maiores que as frações de Fe para todos os solos em estudo, em conseqüência da maior quantidade de $\mathrm{Al}_{2} \mathrm{O}_{3}$ em relação à quantidade de $\mathrm{Fe}_{2} \mathrm{O}_{3}$ obtida pelo ataque sulfúrico. $\mathrm{O}$ maior teor em profundidade foi conferido ao $\mathrm{Al}_{\mathrm{d}}$, o que demonstra o efeito depressor da matéria orgânica sobre a cristalização dos compostos de $\mathrm{Al}$ e a origem do $\mathrm{Al}_{\mathrm{d}}$ a partir dos óxidos e oxi-hidróxidos cristalinos de Fe com alta substituição do Fe por Al (Eusterhues et al., 2005; Inda Júnior et al., 2005). Os dados da extração com oxalato de amônio dos dois elementos, bem como a relação entre as formas extraídas pelos dois extratores (menor que 1), refletiram o maior grau de cristalinidade dos compostos de $\mathrm{Fe}$ e de $\mathrm{Al}$ no material latossólico.

Os valores do PESN variaram de 3,9 a 6,2 unidades de $\mathrm{pH}$, com valor médio de 3,9 em superfície e de 6,0 em subsuperfície, o que se deve possivelmente à redução dos teores de matéria orgânica em profundidade e ao aumento dos teores de óxidos de $\mathrm{Fe}$ e $\mathrm{Al}$.

A equação de regressão do PESN em função do teor de $\mathrm{C}$ orgânico e $\mathrm{Al}_{0}(\mathrm{PESN}=5,582$ - 0,160 $\mathrm{COT}+0,128 \mathrm{Al}_{0}$ ) demonstrou que o teor de $\mathrm{C}$, de forma inversa, e o teor de Al extraído com oxalato de amônio, de forma direta, explicam a quase totalidade $\left(\mathrm{R}^{2}=\right.$ $0,9865)$ da variação do PESN dos solos estudados. Considerando que o teor de $\mathrm{Al}_{0}$ foi menor que os teores dos componentes cristalinos nos solos, esse resultado reitera a elevada reatividade desse composto e a sua importância no comportamento eletroquímico dos solos, tendo em vista sua elevada superfície específica (SE) (Hodson et al., 1998). 
De acordo com Neter et al. (1990), a multicolinearidade existe quando variáveis independentes da equação de regressão múltipla apresentam grau acentuado de correlação e quando os valores do fator de inflação da variável (VIF) forem maiores que 10. Na equação de regressão do PESN foi comprovada a ausência de multicolinearidade, indicada pelos valores de VIF menores que 10, o que reforça a significância estatística e o significado físico da influência do teor de $\mathrm{C}$ orgânico e de óxidos de $\mathrm{Al}$ mal cristalizados sobre o PESN.

Algumas correlações foram feitas entre o PESN e os atributos químicos dos solos estudados. O COT apresentou correlação inversa com o $\operatorname{PESN}(r=-0,96$, $\mathrm{p}<0,01, \mathrm{n}=6$ ), evidenciando a importância da matéria orgânica no manejo de solos mais intemperizados, conforme Chaves \& Trajano (1992). A carga proveniente da matéria orgânica tem origem principalmente nos grupos carboxílicos, hidroxílicos, fenólicos, quinônicos e enólicos (Nkhalamba et al., 2003), os quais contribuem para a carga negativa. Essa contribuição da carga negativa pela matéria orgânica é mais pronunciada em solos oxídicos dos trópicos úmidos, reduzindo o $\mathrm{PCZ}$ desses solos em uma unidade de $\mathrm{pH}$ para cada $1 \mathrm{dag} \mathrm{kg}^{-1}$ de aumento do teor de C orgânico total (Gillman, 1985).

O PCZ estimado pela equação de Keng \& Uehara (1974) mostrou-se adequado para estimar o PESN a partir dos dados de $\mathrm{pH}$ desses solos, pois os valores obtidos a partir dessa equação mostraram-se significativamente correlacionados com o PESN obtido a partir da titulação potenciométrica $(\mathrm{r}=0,97$, $\mathrm{p}<0,01, \mathrm{n}=6$ ). Resultados semelhantes foram obtidos por Alves (2002), Silva et al. (1996) e Siqueira et al. (1990).

Destacam-se os elevados valores de PESN obtidos nos horizontes $B_{\mathrm{w}}$ dos perfis 1 e 3 , evidenciando predomínio de cargas elétricas positivas, confirmado pelos valores positivos do $\Delta \mathrm{pH}$ e pela carga elétrica líquida dos solos.

O índice $\Delta \mathrm{pH}$ mostrou-se altamente correlacionado com a carga elétrica líquida $\left(\mathrm{PESN}-\mathrm{pH} \mathrm{H}_{2} \mathrm{O}\right)(\mathrm{r}=$ $0,99, \mathrm{p}<0,01, \mathrm{n}=6$ ), demonstrando a aplicabilidade desse índice na determinação da carga elétrica líquida do solo. Assim, observou-se que, nas três concentrações de eletrólito na titulação potenciométrica, valores positivos de carga elétrica líquida somente apareceram nas amostras do horizonte $\mathrm{B}$, onde o efeito da matéria orgânica, no sentido de abaixar o ponto de efeito salino nulo e gerar cargas negativas, foi menor.

O PESN apresentou correlação positiva e significativa com os teores de $\mathrm{Fe}_{0}$ e $\mathrm{Al}_{0}(\mathrm{r}=1, \mathrm{p}<0,01$, $\mathrm{n}=6$ ), demonstrando que os maiores valores de PESN estão relacionados a maiores quantidades desses óxidos nos solos. Essa correlação acentuada também foi observada por Alves (2002), Perez et al. (1993) e Sakurai et al. (1989), o que reitera a importante atuação dos óxidos na elevação do PESN em subsuperfície. Houve correlação positiva entre o PESN e os teores de Fe $(\mathrm{r}=0,65)$ e $\mathrm{Al}(\mathrm{r}=0,96)$ cristalizados $\left(\mathrm{Fe}_{\mathrm{d}}\right.$ e $\left.\mathrm{Al}_{\mathrm{d}}\right)$ em horizontes $\mathrm{B}$ dos solos da toposseqüência, indicando que a alta taxa de cristalização dos óxidos de Fe está relacionada à idade do solo e, conseqüentemente, ao seu estádio de intemperismo e a valores de PESN elevados.

\section{CONCLUSÕES}

1. Os teores dos óxidos de $\mathrm{Al}$ de baixa cristalinidade e de $\mathrm{C}$ orgânico total exerceram maior influência na definição do PESN para os solos estudados.

2. O PCZ obtido a partir dos dados de $\mathrm{pH}$ dos solos mostrou-se adequado para estimar o PESN.

3. Os solos da toposseqüência apresentaram inversão de carga em profundidade devido à maior influência da fração mineral dos solos.

\section{LITERATURA CITADA}

AGBENIN, J.O. \& RAIJ, B. van. Rate processes of calcium, magnesium and potassium desorption from variablecharge soils by mixed ion exchange resins. Geoderma, 93:141-157, 1999.

ALLEONI, L.R.F. \& CAMARGO, O.A. Mineralogia da fraçao argila desferrificada de Latossolos ácricos. Sci. Agríc., 53:416-421, 1995.

ALLEONI, L.R.F. \& CAMARGO, O.A. Modelos de dupla camada difusa de Gouy-Chapman e Stern aplicados a Latossolos ácricos paulistas. Sci. Agric., 51:315-320, 1994a.

ALLEONI, L.R.F. \& CAMARGO, O.A. Potencial elétrico superficial e carga elétrica líquida de Latossolos ácricos. R. Bras. Ci. Solo, 18:181-185, 1994b.

ALVES, M.E.; MACEDONIO, C.R. \& LAVORENTI, A. Ponto de efeito salino nulo: Determinação analíticocomputacional a partir de dados de titulação potenciométrica. R. Bras. Ci. Solo, 24:553-559, 2002.

ALVES, M.E. Atributos mineralógicos e eletroquímicos, adsorção e dessorção de sulfato em solos paulistas. Piracicaba, Escola Superior de Agricultura "Luiz de Queiroz”, 2002. 154p. (Tese de Doutorado)

BARREAL, M.E.; ARBESTAIN, M.C. \& MACÍAS, F. Chemical properties and soil color of some Oxisols from Brazil and Spain in relation to sulfate sorption. Soil Sci., 168:718$729,2003$.

CAMARGO, O.A.; MONIZ, A.C.; JORGE, J.A. \& VALADARES, J.M.A.S. Métodos de análise química, mineralógica e física de solos do Instituto Agronômico de Campinas. Campinas, Instituto Agronômico, 1986. 94p. (Boletim Técnico, 106)

CANELLAS, L.P.; BERNER, P.G.; SILVA, S.G.; SILVA, M.B. \& SANTOS, G.A. Frações da matéria orgânica em seis solos de uma toposseqüência no Estado do Rio de Janeiro. Pesq. Agropec. Bras., 35:133-143, 2000. 
CHAVES, L.H.G. \& TRAJANO, M.D.M. Determinação do ponto de carga zero e das cargas elétricas do horizonte Ap de solos do Estado da Paraíba. R. Bras. Ci. Solo, 16:415418, 1992.

CHOROVER, J. \& SPOSITO, G. Surface charge characteristics of kaolinitic tropical soils. Geochim. Cosmochim. Acta, 59:875-884, 1995.

CHUAN-WEN, P.; WANG, M.; ZHUANG, S. \& KING, H. Free and non-crystalline $\mathrm{Fe}$-oxides to total iron concentration ratios correlated with ${ }^{14} \mathrm{C}$ ages of three forest soils in central Taiwan. Soil Sci., 169:582-589, 2004.

COSCIONE, A.R.; MONIZ, A.C.; PÉREZ, D.V.; FERREIRA, M.M.C. \& CAMARGO, O.A. Chemical and electrochemical properties of an Oxisol-Ultisol transition in the state of São Paulo, Brazil. Geoderma, 34:375-388, 2005 .

COSTA, L.M.; MORAIS, E.J.; RIBEIRO, A.C. \& FONSECA, S. Cargas elétricas de um Latossolo Vermelho-Amarelo com diferentes coberturas florestais. R. Ceres, 177:351-359, 1984.

EMPRESA BRASILEIRA DE PESQUISA AGROPECUÁRIA EMBRAPA. Sistema brasileiro de classificação de solos. Brasília, Sistema de Pesquisa da Informação, 1999. 412p.

EUSTERHUES, K.; RUMPEL, C. \& GEL-KNABNER, I.K. Organo-mineral associations in sandy acid forest soils: Importance of specific surface area, iron oxides and micropores. Eur. J. Soil Sci., 56:753-763, 2005.

FONTES, M.P.F.; CAMARGO, O.A. \& SPOSITO, G. Eletroquímica das partículas coloidais e sua relação com a mineralogia de solos altamente intemperizados. Sci. Agric., 58:627-646, 2001.

GALLEZ, A.; HERBILLON, A.J. \& JUO, A.S.R. Characteristics of silica sorption and solubility as parameters to evaluate the surface properties of tropical soils: I. The index of silica reactivity. Soil Sci. Soc. Am. J., 41:1146-1150, 1977.

GHIDIN, A.A.; MELO, V.F.; LIMA, V.C. \& COSTA LIMA, J.M.J. Oxisol toposequences developed from basaltic rocks in Paraná State, Brazil: II - Relationship between clay fraction mineralogy and physical soil properties. R. Bras. Ci. Solo, 30:345-357, 2006.

GILLMAN, G.P. Influence of organic matter and phosphate content on the point of zero charge of variable charge components in oxidic soils. Austr. J. Soil Res., 23:643-646, 1985.

HENDERSHOT, W.H. \& LAVKULICH, L.M. The use of zero point of charge (ZPC) to assess pedogenic development. Soil Sci. Soc. Am. J., 42:468-472, 1978.

HENDERSHOT, W.H.; SINGLETON, G.A. \& LAVKULICH, L.M. Variation in surface charge characteristics in a soil chronosequence. Soil Sci. Soc. Am. J., 43:387-389, 1979.

HODSON, M.E.; LANGAN, S.J.; KENNEDY, F.M. \& BAIN, D.C. Variation in soil surface area in a chronosequence of soils from Geln Feshie, Scotland and its implications for mineral weathering rate calculations. Geoderma, 85:1$18,1998$.
HSU, P.H. Aluminum hydroxides and oxyhydroxides. In: DIXON, J.B. \& WEED, S.B., eds. Minerals in soil environments. Madison, Soil Science Society of America, 1989. v.7, p.331-378.

INDA JUNIOR, A.V. \& KÄMPF, N. Avaliação de procedimentos de extração dos óxidos de ferro pedogênicos com ditionitocitrato-bicarbonato de sódio. R. Bras. Ci. Solo, 27:1139-1147, 2003.

INDA JUNIOR, A.V.; KÄMPF, N.; GIASSON, E. \& SILVA, J.A.L. Cinética da dissolução redutiva da goethita e hematita em solos poligenéticos. Ci. Rural, 35:553-561, 2005 .

JONES, R.C. X-Ray diffraction line profile analysis phosphorus sorption by 11 Puerto Rico soils. Soil Sci. Soc. Am. J., 45:818-825, 1981.

KÄMPF, N. \& SCHWERTMANN, U. Quantitative determination of goethite and hematite in kaolinites soils by x-rays diffraction. Clay Min., 17:359-363, 1982.

KÄMPF, N. \& CURI, N. Argilominerais em solos brasileiros. In: CURI, N.; MARQUES, J.J.G.S.M.; GUILHERME, L.R.G.; LIMA, J.M.; LOPES, A. S.; ALVAREZ V., V.H. Tópicos em ciência do solo. Viçosa, MG, Sociedade Brasileira de Ciência do Solo, 2003. v.3. p.1-54.

KENG, J.C.W. \& UEHARA, G. Chemistry, mineralogy and taxonomy of Oxisols and Ultisols. Proc. Soil Crop Sci. Soc., 33:119-126, 1974.

KER, J.C. \& RESENDE, M. Caracterização química e mineralógica de solos brunos subtropicais do Brasil. R. Bras. Ci. Solo, 14:215-225, 1990.

LACERDA, M.P.C.; ANDRADE, H. \& QUÉMÉNEUR, J.J.G. Correlação entre material de origem e perfis de alteração em solos com B textural na região de Lavras, MG. Ci. Agrotecnol., 24:585-596, 2000.

MEHRA, O.P. \& JACKSON, M.L. Iron oxide removal from soils and clays by dithionite-citrate systems buffered with sodium bicarbonate. Clays Clay Miner., 7:317-327, 1960.

MUGGLER, C.C.; van LOEF, J.J.; BUURMAN, P. \& van DOESBURG. J.D.J. Mineralogical and (sub) microscopic aspects of iron oxides in polygenetic Oxisols from Minas Gerais, Brazil. Geoderma, 100:147-171, 2001.

NASCIMENTO, R.A.M.; CUNHA, L.H. \& RAMOS, D.P. Comparações entre o ponto de carga zero (titulação potenciométrica), mineralogia e diversos outros parâmetros para quatorze perfis de Latossolos. In: REUNIÃO DE CLASSIFICAÇÃO, CORRELAÇÃO DE SOLOS E INTERPRETAÇÃO DE APTIDÃO AGRÍCOLA, Rio de Janeiro, 1984. Anais. Rio de Janeiro, Serviço Nacional de Levantamento e Conservação de Solos, 1988. v.3. p.365-390.

NETER, J.; WASSEMAN, W. \& KUTNER, M.H. Applied linear statical models: Regression, analysis of variance and experimental designs. Boston, IRWIN, 1990. 1181p.

NKHALAMBA, J.W.; ROWELL, D.L. \& PILBEAN, C.J. The development and contribution of surface charge by crop residues in two Malawian acid soils. Geoderma, 115:281302,2003 
NORRISH, K. \& TAYLOR, R.M. The isomorphous replacement of iron by aluminium in soil goethites. J. Soil Sci., 12:294304, 1961.

PAI, C.; WANG, M. \& CHIU, C. Clay mineralogical characterization of a toposequence of perhumid subalpine forest soils in northeastern Taiwana. Geoderma, 138:177$184,2007$.

PARKER, J.C.; ZELAZNY, L.W.; SAMPATH, S. \& HARRIS, W.G. A critical evaluation of the extension of zero point of charge (ZPC). Theory to soil systems. Soil Sci. Soc. Am. J., 43:668-673, 1979.

PÉREZ, D.V.; RAMOS, D.P.; NASCIMENTO, R.A.M. \& BARRETO, W.O. Propriedades eletroquímicas de horizontes B texturais. R. Bras. Ci. Solo, 17:157-164, 1993.

RAIJ, B.van \& PEECH, M. Electrochemical properties of some Oxisols and Alfisols of the tropics. Soil Sci. Soc. Am. Proc., 36:587-593, 1972.

RESENDE, M. \& SANTANA, D.P. Uso das relações Ki e Kr na estimativa da mineralogia para a classificação dos Latossolos. In: REUNIÃO DE CLASSIFICAÇÃO, CORRELAÇÃO DE SOLOS E INTERPRETAÇÃO DE APTIDÃO AGRÍCOLA, Rio de Janeiro, 1988. Anais. Rio de Janeiro, Embrapa, SNLCS, SECS, 1988. v.3. p.225232 .

SAKURAI, K.; OHDATE, Y. \& KYUMA, K. Factors affecting zero point of charge (ZPC) of variable charge soils. Soil Sci. Plant Nutr., 35:21-31, 1989.

SAS Institute. SAS: Principles of regressions analysis - course notes. Cary, 1994. 655p.

SCHWERTMANN, U. Some properties of soil and synthetic iron oxides. In: STUCKI, J.W.; GOODMAN, B.A. \& SCHWERTMANN, U., eds. Iron in (ours) soils and clay minerals. Dordrecht, Reidel, 1988. p.203-250.
SCHWERTMANN, U. The differentiation of iron oxides in soils by a photochemical extraction with acid ammonium oxalate. Zeitschrift Pflanzenernaehrung Bodenk., 105:194-201, 1964.

SCHWERTMANN, U. \& CARLSON, L. Aluminum influence on iron oxides: XVII. Unit-cell parameters and aluminum substitution of natural goethites. Soil Sci. Soc. Am. J., 58:256-261, 1994

SCHWERTMANN, U. \& KÄMPF, N. Properties of goethite and hematite in kaolinitic soils of southern and central Brazil. Soil Sci., 139:344-350, 1985.

SCHWERTMANN, U. \& TAYLOR, R.M. Iron oxides. In: DIXON, J.B. \& WEED, S.B., eds. Minerals in soil environments. 2.ed. Madison, Soil Science Society of America, 1989. p.379-438.

SILVA, M.L.N.; CURI, N.; MARQUES, J.J.G.S.M.; GUILHERME, L.R. \& LIMA, J.M. Ponto de efeito salino nulo e suas relações com propriedades mineralógicas e químicas de Latossolos brasileiros. Pesq. Agropec. Bras., 31:663-671, 1996.

SIQUEIRA, C.; LEAL, J.R.; VELLOSO, A.C.X. \& SANTOS, G.A. Eletroquímica de solos tropicais de carga variável: II Quantificação do efeito da matéria orgânica sobre o ponto de carga zero. R. Bras. Ci. Solo, 14:13-17, 1990.

SPOSITO, G. The chemistry of soils. New York, Oxford University Press, 1989. 277p.

UEHARA, G. \& GILLMAN, G.P. The mineralogy, chemistry and physics of tropical soils with variable charge clays. Boulder: Westview Press, 1981. 170p.

ZHANG, X.N. \& ZHAO, A.Z. Surface charge. In: YU, T.R., eds. Chemistry of variable charge soils. New York, Oxford University Press, 1997. p.17-63. 